ICNMM2017-5536

\title{
MODELING OF AN AC-ELECTRO-OSMOSIS BASED MICROFLUIDIC MIXER
}

\section{H. Dilara Uslu}

Microfluidics \& Lab-on-a-chip Res. Group

Mechanical Engineering Department

I.D. Bilkent University

Ankara 06800 TURKEY

Email: dilara.uslu@bilkent.edu.tr

\author{
Çetin Canpolat
}

\author{
Biomedical Eng. Dept. \\ Çukurova University \\ Adana 01330 TURKEY \\ Email: ccanpolat@cu.edu.tr
}

\author{
Barbaros Çetin* \\ Microfluidics \& Lab-on-a-chip Res. Group \\ Mechanical Engineering Department \\ I.D. Bilkent University \\ Ankara 06800 TURKEY \\ Email: barbaros.cetin@bilkent.edu.tr
}

\section{ABSTRACT}

The purpose of this study is presenting an active micromixer, which is based on AC electro-osmotic flow driven on $3 D$ micro wires. In order to solve governing equations of AC electroosmosis, a commercial software COMSOL Multiphysics ${ }^{\circledR}$ is implemented. Different wire configurations with various imposed electric fields and flow rates are tested for evaluating mixing efficiencies. The analyses show that mixing performance is significantly improved by number of the wires as well as wire orientation. It is also revealed that the degree of mixing can also be controlled by the tuning of the applied voltage for a given flow rate.

\section{NOMENCLATURE}

$C_{D L}$ capacitance per unit area across the double layer

$D$ diffusion coefficient

$V_{p p} \quad$ peak-to-peak voltage

$P e$ Peclet number

$P$ pressure

$q_{D L} \quad$ charge per unit area in the double layer

Re Reynolds number

$V_{j} \quad$ voltage applied to the electrode $\mathrm{j}$

$\partial \phi_{D L}$ potential drop in the double layer

$c$ concentration

$\partial \phi_{d}$ potential drop in the diffuse layer

*Address all correspondence to this author. $\rho$ density

$\mu$ viscosity

$\sigma$ conductivity of electrolyte

$\omega$ frequency

$\varepsilon_{r}$ relative permittivity

$\varepsilon_{0} \quad$ vacuum permittivity

$\lambda_{D} \quad$ Debye length

$\Lambda$ ratio of potential drop in the diffuse layer to the potential in the double layer

$\eta_{m} \quad$ mixing efficiency

\section{INTRODUCTION}

Microfluidic systems are used in many applications like synthesis, chemical reactions, cell separation and characterization. Microfluidics is not simply a miniaturized version of macrofluidic systems since some terms, such as surface tension, diffusion and viscous forces dominate the flow field [1]. Typically in microfluidic applications, Reynolds number $(R e)$ is sufficiently small to constitute Stokes flow regime, in which the fluid particles follow the flow boundaries; hence, mixing becomes challenging. When mixing comes into prominence in microfluidics, Peclet number $(\mathrm{Pe})$, which gives the proportion of advection to diffusion, reduces below unity. In low $R e$ and $P e$, mixing is mainly dominated by molecular diffusion and molecular diffusion coefficient, $(D)$, is $10^{-9} \mathrm{~m}^{2} / \mathrm{s}$ in most of the microfluidic applications [2]. Therefore, it is hard to mix fluids with such a 
low $D$ and this situation requires relatively high channel length and long time period, which do not provide feasible solution for many microfluidic applications. To speed up micro-mixing process, many different methods have been proposed in the literature, and these mixers are mainly classified as passive and active micro-mixers.

Passive mixers simply utilize the geometry of the microchannel and requires no external energy input. Passive micromixers are designed such that the diffusion length, required time are minimized and the interface between two fluids is enlarged [1]. Different geometries such as zigzag [3] and serpentine [4] microchannels have been proposed. To improve mixing, obstacles are also located inside the channel [5]. Active micro-mixers disturb the stable fluid flow and produce perturbations by applying external energy, which in turn speeds up diffusion process and also reduces diffusion time. The external energy methods implemented on active micro-mixers are categorized as; pressure disturbance [6], dielectrophoretic [7,8], acoustic/ultrasonic [9,10], magnetic actuation [11] and electrokinetic [12]. Although more control parametres in active mixing, such as the external field strength, the flow rate and the channel geometry increase the complexity, these parameters enable the control of the mixing more precisely and the enhancement for mixing efficiency. Among these techniques, electrokinetic mixers are quite popular since electrokinetic techniques are well-developed for many microfluidic applications [13]. In this phenomena, electrokinetic instability is created and the interface between two fluids are stretched and folded so that this behavior induces mixing inside the channels. In most of the studies in the literature, planar electrodes with very low separation distance are used by placing them on the walls of the channel in ac electro-osmosis. Electric field is created by applying voltage directly to the electrodes, mixing chambers are created. Chen et al. [14] presented a micromixer having electrodes on the walls of the mixing chamber and they reached 95\% mixing efficiency. Oddy et al. [15] offerred Yshaped microchannel having 2 ports for applying ac electric field. By using electrokinetic instability, fluids are mixed within very small space called as mixing chamber [15]. Another way of generating small scale vortices is induced-charge electro-osmosis (ICEO) phenomena. In this case, cylindrical obstacles, in which electric field is not applied directly to the conductive rods, are located vertically inside the microchannel. ICEO is nonlinear electrokinetic phenomenon, which exhibits quadrupolar flow structure with four microvortices around cylindrical conductive material [16]. ICEO flow also increases the degree of vorticity in the close field of conducting material [16].Therefore, it provides an alternative tool for micromixing applications. In Wu's study [17], conducting hurdles having certain height are placed on the walls of the microchannel symmetrical to the flow direction. By applying potential difference between inlet and outlet of the channel, mixing near these hurdles with varying flow rates and electric fields is achieved. Although the use of planar elec-

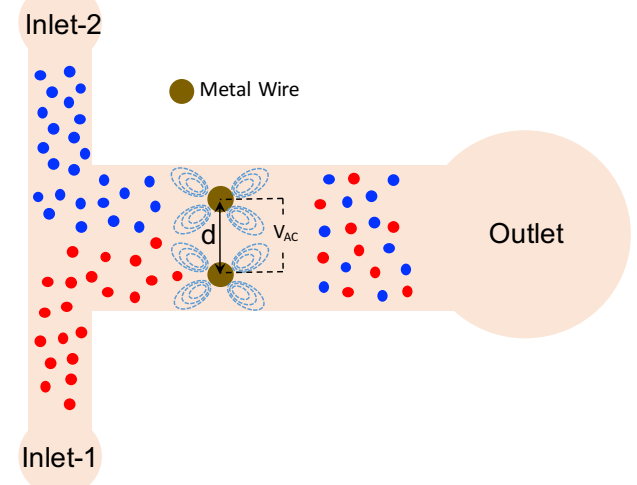

FIGURE 1. SCHEMATICS OF THE PROBLEM

trodes for microfluidic applications is common for the ease of fabrication, the EO flow is confined in the vicinity of the electrode surface. On the other hand, the use of 3D electrodes can enhance the mixing performance since region where the EO flow is effective can be extended. Recently, researchers introduced different techniques to embed 3D electrodes into the microchannels $[13,18,19]$.

In this study, a computational modeling of a micro-mixer based on ac electro-osmotic flow driven on 3D micro wires is presented. The flow is pressure driven and vortexes are generated within the microchannel around the micro-wires strategically located inside the microchannel. With the developed model, different orientations of micro-wires are assessed in terms of mixing efficiency as a function of applied voltage and the flow rate.

\section{ANALYSIS}

The schematic drawing of the problem is given in Fig. 1 . The microfluidic device has two inlets and one outlet. In the main channel metal wires are located. In this schematic drawing, the simplest case is given. Two and four wire configurations are also analyzed. The main channel has a width of $1500 \mu \mathrm{m}$. The diameter of the wires are $200 \mu \mathrm{m}$ with a center-to-center separation (d) of $700 \mu \mathrm{m}$ and $500 \mu \mathrm{m}$. The fabrication of the device is planned to be performed by high-precision mechanical machining, therefore the dimensions are selected to ensure non-problematic fabrication. The flow field is governed by incompressible Navier-Stokes equation,

$$
\begin{aligned}
\nabla \cdot(\rho \mathbf{u}) & =0 \\
\rho(\mathbf{u} \cdot \nabla) \mathbf{u} & =-\nabla p+\mu \nabla^{2} \mathbf{u}+\rho \mathbf{g}
\end{aligned}
$$

with the no-slip boundary conditions at the channel walls, specified flow rates at the reservoirs and zero pressure boundary condition at the exit. 
An ac electric field is applied on the metal wires, this field generates AC-EOF on the electrode surface. When electric field is applied around polarizable objects immersed in electrolyte solution, oppositely charged ions in electrical double layer (EDL) are induced around these objects and $\zeta$ potential changes [20]. Due to non-zero current, conductive object creates a thin layer of positively charged ion on one side and negatively charged ions on the other side and so, there are equal amounts of surface charges induced around the objects as well. When the conductor becomes fully-polarized, the conductor behaves like an insulator and it reaches steady-state condition. The electric field inside the microchannel can be modeled by Laplace equation:

$$
\nabla^{2} \phi=0
$$

Outside of the EDL, the electric potential on the electrode surface is given in Green et al. [21] for 1D case. For 3D applications, electric potential on the electrode surface is defined as

$$
\sigma \frac{\partial \phi}{\partial \mathbf{n}}=i \omega q_{D L}
$$

where $\sigma$ is the electrical conductivity of electrolyte and $q_{D L}$ is the charge per unit area in the EDL [21]. By the assumption of very small voltage drop across EDL, $q_{D L}$ is taken as $q_{D L}=$ $C_{D L}\left(\phi-V_{j}\right)$ in Green et al. [21].

$$
\sigma \frac{\partial \phi}{\partial \mathbf{n}}=i \omega C_{D L}\left(\phi-V_{j}\right)
$$

where $C_{D L}$ is the capacitance per unit area across the EDL, $\omega$ is the applied frequency and $V_{j}$ is the applied voltage on the electrode $j$ [21]. Based on Debye-Huckel theory, the double layer capacitance $\left(C_{D L}\right)$ can be approximated as $\varepsilon / \lambda_{D}$ [21]. Here, $\lambda_{D}$ is the Debye length, which defines the length of the double layer. Using the thin EDL approximation, the slip-velocity on the electrode surface can be written as [22]:

$$
\mathbf{u}=-\frac{\varepsilon}{4 \mu} \Lambda \frac{\partial}{\partial \mathbf{n}}\left|\phi-V_{j}\right|^{2}
$$

where $\mu$ is the viscocity and $\Lambda$ is a ratio of the potential drop in the diffuse layer to the potential drop in the whole EDL $\left(\Delta \phi_{d} / \Delta \phi_{D L}\right)$. For the analysis, $\Lambda$ is assumed as 1.0. In Green's study [21], $\Lambda$ value is taken as 1.0 because of the Debye-Huckel theory of very small double layer approximation. However, based on the experimental data, $\Lambda$ is slightly changed to match experimental data with numerical data because in real cases, the capaticance of the double layer does not depend on only diffuse layer but it also depends on compact layer [21].
The concentration field is governed by convection-diffusion equation,

$$
\mathbf{u} \cdot \nabla c=D \nabla^{2} c
$$

subjected to insulated boundary at the channel walls and the specified concentration at the reservoirs. Herein, $c$ represents the dimensionless mole fraction of the acetic acid. Therefore, $c$ is assigned as unity at the inlets of acetic acid, and assigned as zero for the inlets of water. At the exit, convective flux boundary condition is assigned.

In order to quantify the mixing performance, the mixing efficiency at the exit of the mixing section is determined. Mixing efficiency can be determined as [14],

$$
\eta_{m}=1-\frac{\int_{A}\left|c-c_{\infty}\right| d A}{\int_{A}\left|c_{o}-c_{\infty}\right| d A}
$$

where $c$ is the sample concentration, and $c_{o}$ and $c_{\infty}(=0.75)$ are the sample concentrations in the completely unmixed and completely mixed conditions, respectively. Therefore, $\eta_{m}=0$ indicates a completely unmixed state, and $\eta_{m}=1.0$ indicates complete mixing.

\section{COMPUTATIONAL MODELING}

For computation, electric currents (ec), laminar flow (spf) and transport of diluted species (tds) modules of COMSOL Multiphysics ${ }^{\circledR}$ are used. In ec module, the parameters needed to solve electric potential are identified as follows; relative permittivity $\left(\varepsilon_{r}\right)$ is 80 , vacuum permittivity $\left(\varepsilon_{0}\right)$ is $8.854 \times 10^{-8} \mathrm{~F} / \mathrm{m}$, Debye length ( $\left.\lambda_{D}\right)$ is $4.858 \mathrm{~nm}$ conductivity of the liquid $(\sigma)$ is $6 \mathrm{mS} / \mathrm{cm}$ and frequency $(\omega)$ is $100 \mathrm{kHz}$. Different voltage values between $0 \mathrm{~V}$ to $125 \mathrm{~V}$ are applied to the wires. The velocity field parameters that are deficient in spf module are defined as follows; viscosity $(\mu)$ is $10^{-3} \mathrm{~Pa} \cdot \mathrm{s}$, density $(\rho)$ is $1000 \mathrm{~kg} / \mathrm{m}^{3}$. As flow rates, $5 \mathrm{~mL} / \mathrm{h}, 1.0 \mathrm{~mL} / \mathrm{h}$ and $0.02 \mathrm{~mL} / \mathrm{h}$ are applied. For concentration field, non-dimensional concentration of unity and zero is assigned from the inlets.

Strictly speaking, a pressure driven flow coupled with an AC-EOF around 3D metal electrodes is 3D and the concentration field is $3 \mathrm{D}$ as well. However, the purpose of this study is to assess the different electrode configuration in terms of the mixing performance. Therefore, as an initial study, a $2 \mathrm{D}$ model is compared to a full 3D model. The thickness in 3D model is determined as $100 \mu \mathrm{m}$ but at the top surface of the model, symmetry boundary condition is given. That's, $200 \mu \mathrm{m}$ channel thickness is acquired. By this way, computation time of the simulation is 


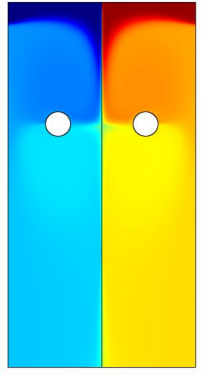

(A) $2 \mathrm{D}\left(\mathrm{V}_{\mathrm{j}}=100 \mathrm{~V}\right)$

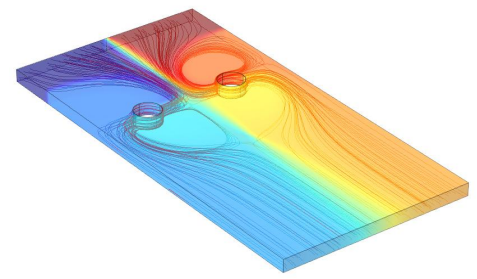

(B) $3 \mathrm{D}\left(\mathrm{V}_{\mathrm{j}}=100 \mathrm{~V}\right)$
FIGURE 2. Comparison between $2 \mathrm{D}$ and 3D modeling

reduced. Quadratic elements are used for electric and concentration field. Element order of P2 + P1 is used for fluid flow. In 2D case, free triangular mesh is used on the interface and around the wires. Three different physics; potential field, velocity field and concentration field are solved separately and the number of degrees of freedom (DOF) values are approximately $315 \mathrm{~K}, 700 \mathrm{~K}$ and $315 \mathrm{~K}$, respectively. In 3D case, a free triangular mesh is defined around the wires for both top and bottom surfaces. Upon applying free triangular mesh, swept mesh is introduced through the channel thickness. DOF is about $1.5 \mathrm{M}$ for the electric field, about $4.5 \mathrm{M}$ for the flow field and $1.5 \mathrm{M}$ for the concentration field. The simulations were performed on a HP Z840 Workstation (Intel Xeon E5-2620, 6-core, 2.40GHz, 128GB RAM). As a first step of the simulations, the electric field is obtained followed by the flow field. At the final step, the concentration field is determined and the mixing efficiency is evaluated when needed. These two simulations are performed for an average inlet velocity of $4.6 \mathrm{~mm} / \mathrm{s}$. Figure 2 shows the concentration field of the two solutions. As seen from the figure, the concentration fields are in good agreement and the two simulations yield almost the same mixing efficiencies which are $64.7 \%$ for 3D case and $67.5 \%$ for $2 \mathrm{D}$ case. This initial study reveals that a $2 \mathrm{D}$ modeling is sufficient to predict the mixing performance, therefore herein after all the simulations are performed in $2 \mathrm{D}$ to reduce the computational load.

\section{RESULTS AND DISCUSSION}

Considering the constraints and the ease of fabrication, four different wire configurations, which are seen in Fig. 3 are tested in this study. The wires have a center-to-center separation of $d$. In the staggered and aligned configuration, the vertical and the horizontal spacing of two consecutive wires are set as $d 1$ and $d$ respectively. Each case is studied with three different flow rates $(0.2 \mathrm{~mL} / \mathrm{h}, 1.0 \mathrm{~mL} / \mathrm{h}$ and $5.0 \mathrm{~mL} / \mathrm{h})$ and distinctive imposed electric field strengths.

Figure 4 shows the 2-wire symmetric case (see Fig. 3-A) for different flow rates and applied voltages. The case for complete
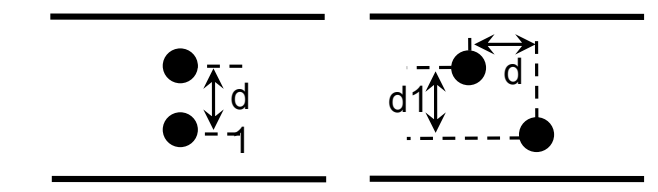

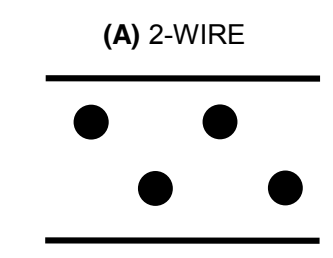

(C) 4-WIRE STAGGERED

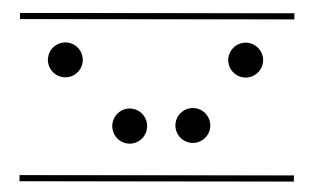

(D) 4-WIRE ALIGNED
(B) 2-WIRE STAGGERED

FIGURE 3. Wire configurations

mixing (red dashed line) and no mixing (black dash line) is also included in the figure. As the voltage increases, the electric field increases and so does the slip-velocity, which results in a vortex with a higher intensity and a better mixing. As seen from the figures the curves are moving from no mixing case to complete mixing case as the voltage increases. The important parameter is the ratio of the pressure driven velocity and the slip-velocity, which is proportional with the applied voltage, on the electrode surface. Inside the channel, the mixing depends on the lateral motion of the species which is dominated by diffusion due to the low Pe number nature of the flow. Therefore, the secondary flow induced around the wires generates convection in the lateral direction and enhances the mixing. As the flow rate decreases, the mixing is better for a given voltage. This can be clearly seen as the two blue curves on Fig. 4-A and Fig. 4-B. As the flow rate is further decreased, similar mixing performance is achieved for lower voltages. As shown from Fig. 4-C, the maximum voltage applied is almost $1 / 3$ of that of $Q=5 \mathrm{~mL} / \mathrm{h}$.

Figure 5 illustrates the mixing efficiency for different wire configurations. The spacing $d$ is taken as $700 \mu \mathrm{m}$ for the first case and for the others, it is defined as $500 \mu \mathrm{m}$. The distance $d 1$ is determined as $700 \mu \mathrm{m}$. The figure shows the results for three different flow rates. The performance of the mixing can be enhanced quite significantly with the wire configuration. First observation is that as the spacing reduced from $700 \mu \mathrm{m}$ to $500 \mu \mathrm{m}$, the electric field strength increases, the intensity of the vortex increases and the mixing efficiency improves for a given voltage. This trend holds for three cases. The 2-wire staggered configuration has a better performance than the 2-wire symmetric case. The reason is that as seen from the streamline given in Fig. 2$\mathrm{B}$, symmetric case generated two symmetric vortexes, and actually penetration of one stream into another is mainly through diffusion. However, in the staggered configuration, one electrode generates a vortex and disturbs the other stream with the lower concentration, and the second wire generates a lateral motion for the already disturbed fluid flow. Implementation of one more set 


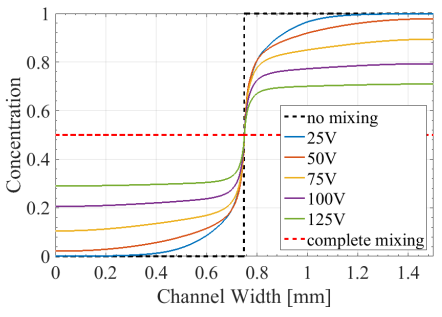

(A) $\mathrm{Q}=5.0 \mathrm{~mL} / \mathrm{h}$

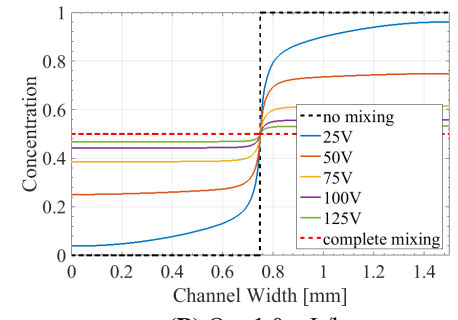

(B) $\mathrm{Q}=1.0 \mathrm{~mL} / \mathrm{h}$

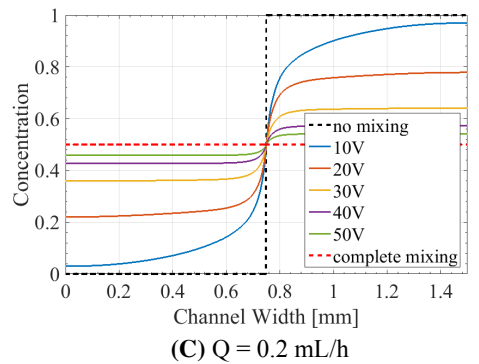

FIGURE 4. Concentration profiles for 2-wire symmetric case at different flow rates and voltages $(d=700 \mu \mathrm{m})$

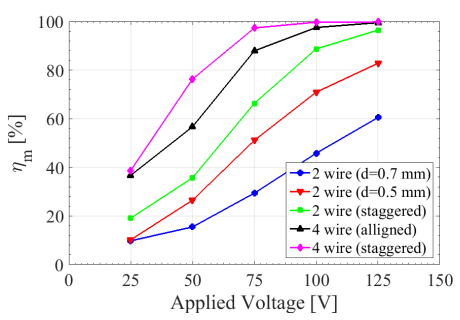

(A) $\mathrm{Q}=5.0 \mathrm{~mL} / \mathrm{h}$

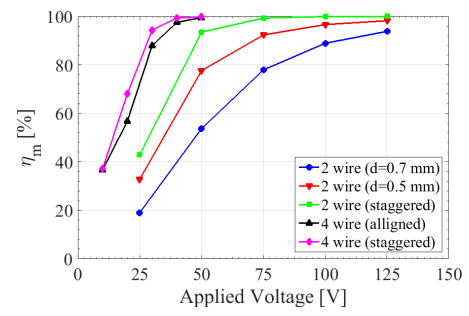

(B) $\mathrm{Q}=1.0 \mathrm{~mL} / \mathrm{h}$

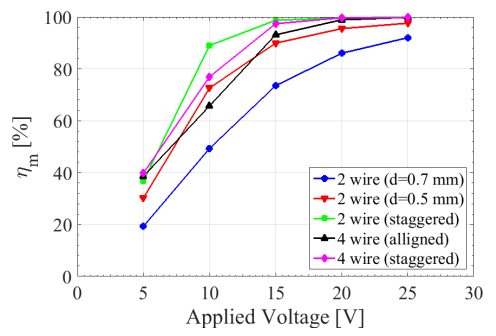

(C) $\mathrm{Q}=0.2 \mathrm{~mL} / \mathrm{h}$

FIGURE 5. Mixing efficiency of different configurations at different flow rates

of staggered electrodes significantly improves the mixing performance. With a relative low voltage, complete mixing is achieved. The 4-wire staggered and aligned configurations give approximately the same performance. Since the disturbance introduced in the channel for both of the configurations is also the same. As the flow rate decreases, the required voltage for complete mixing also drops.

Although the focus of this study is to assess the wire configuration for a better mixing which is usually the goal of the

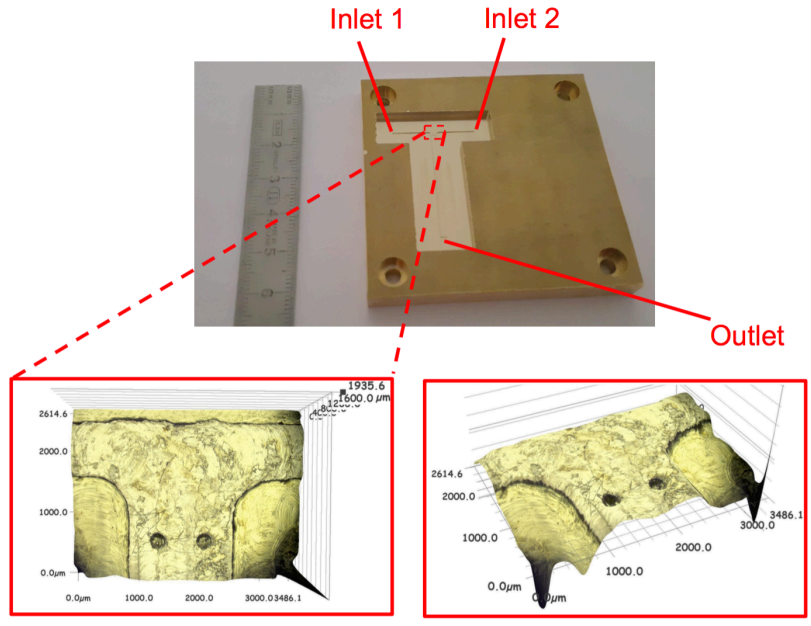

FIGURE 6. Photograph and microscope image of the mold

microfluidic mixer, the results also reveal that the proposed microfluidic mixer can also control the degree of mixing by tuning the voltage for a given flow rate. The flow is driven by pressure difference, and the mixing can be controlled by the voltage which are two separate control parameters. In many of the passive mixers, the flow rate and the degree of mixing is related. The control of the degree of mixing as a separate parameter may be quite important for microfluidic drug delivery applications [23]. The accuracy of the control may be even further improved by applying different voltages for 4 wire configurations, and such a computational model can be an essential tool for the design of such a microfluidic mixer.

For the realization of this microfluidic mixer, the precise alignment of the metal wires inside the microchannel is a critical step. We are planing to achieve this by using high-precision mechanical machining. A preliminary trial has been performed, and a metal mold with holes with the diameter of the metal wires has been fabricated for the center-to-center spacing of $700 \mu \mathrm{m}$. The photograph of the mold is given in Fig. 6. The holes located on the mold will enable the precise positioning of the metal electrodes within the microchannels before the PDMS molding. For the conducting wires commercially available brass and stainless steel wires will be utilized. It is thought that the conductivity of the wire material is significant in terms of double layer capacitance. Therefore, it can be said that capacitance change would affect the mixing performance. To understand this effect, variable materials will also be compared.

\section{CONCLUSIONS}

An AC-EOF based electro-kinetic mixer is presented in this study. The mixing is achieved by the induced AC-EOF around conducting wires located strategically inside the microchannel. 
The effect of the different wire orientations, applied voltage and flow rate on the mixing performance is assessed. It has been shown that the mixing performance can be significantly improved by the number and orientation of the wires. Furthermore, the results also reveal that the degree of mixing can be managed accurately by controlling the applied voltage, which may be quite useful for drug delivery applications.

The essence of the proposed mixer is the 3D strategically and precisely located metal wires inside a microchannel. The fabrication of the structure will be performed using highprecision mechanical machining, which is one of our research group's strong suits. A preliminary mold for the fabrication of the mixer is also presented.The experimental verification of the findings of this study will be our future research direction. We also would like study the effect of material type of the wire and the frequency as a next step.

\section{REFERENCES}

[1] Capretto, L., Cheng, W., Hill, M., and Zhang, X., 2011. Micromixing Within Microfluidic Devices. Springer Berlin Heidelberg, Berlin, Heidelberg.

[2] Cetin, B., Asik, M. D., and Taze, S., 2013. "Design and fabrication of a microfluidic device for synthesis of chitosan nanoparticles". J. Nanotechnol. Eng. Med., 4, p. 031004.

[3] Mengeaud, V., Josserand, J., and Girault, H. H., 2002. "Mixing processes in a zigzag microchannel: finite element simulations and optical study". Anal. Chem., 74(16), pp. 4279-4286.

[4] Liu, R. H., Stremler, M. A., Sharp, K. V., Olsen, M. G., Santiago, J. G., Adrian, R. J., Aref, H., and Beebe, D. J., 2000. "Passive mixing in a three-dimensional serpentine microchannel”. J. Microelectromech. Syst., 9(2), pp. 190197.

[5] Jonhson, T., Ross, D., and Locascio, L., 2002. "Rapid microfluidic mixing”. Anal. Chem., 74(1), pp. 45-51.

[6] Niu, X., and Lee, Y. K., 2003. "Efficient spatial-temporal chaotic mixing in microchannels". J. Micromech. Microeng., 13, April, pp. 454-462.

[7] Choi, E., Kim, B., and Park, J., 2009. "High-throughput microparticle separation using gradient travelling wave dielectrophoresis". J. Micromech. Microeng., 19, pp. 454462.

[8] Deval, J., Tabeling, P., and Ho, C. M., 2002. "A dielectrophoretic chaotic mixer". In IEEE International Conference. Fifteenth IEEE International Conference on Micro Electro Mechanical Systems, pp. 36-39.

[9] Liu, R. H., Lenigk, R., Druyor-Sanchez, R. L., Yang, J., and Grodzinski, P., 2003. "Hybridization enhancement using cavitation microstreaming”. Anal. Chem., 75, pp. 19111917.

[10] Yaralioglu, G. G., Wygant, I. O., Marentis, T. C., and
Khuri-Yakub, B. T., 2004. "Ultrasonic mixing in microfluidic channels using integrated transducers". Anal. Chem., 76, April, pp. 3694-3698.

[11] Choi, E., Kim, B., and Park, J., 2011. "Mathematical modelling and analysis of a magnetic nanoparticle-enhanced mixing in a microfluidic system using time-dependent magnetic field". IEEE Trans. Nanotechnol., 10, September.

[12] Lee, C. Y., Chang, C. L., Wang, Y. N., and Fu, L. M., 2011. "Microfluidics mixing: A review". Int. J. Mol. Sci., 12(5), pp. 3263-3287.

[13] Cetin, B., Ozer, M. B., and Solmaz, M. E., 2014. "Microfluidic bio-particle manipulation for biotechnology". Biochem. Eng. J., 92, pp. 63-82.

[14] Chen, C. K., and Cho, C. C., 2008. "Electrokinetically driven flow mixing utilizing chaotic electric fields". $\mathrm{Mi}$ crofluidics and Nanofluidics, 5(6), pp. 785-793.

[15] Oddy, M., Santiago, J., and Mikkelsen, J., 2001. "Electrokinetic instability micromixing". Anal. Chem., 73, pp. 58225832.

[16] Canpolat, C., Qian, S., and Beskok, A., 2014. “'inducedcharge electro-osmosis of polymer-containing fluid around a metallic rod". Microfluidics and Nanofluidics, 16(1), pp. 247-255.

[17] Wu, Z., 2008. "Nonlinear Induced-Charge Electrokinetics". $\mathrm{PhD}$ Thesis, Vanderbilt University, Nashville, Tennessee, December.

[18] Li, M., Li, W. H., Zhang, J., Alici, G., and Wen, W., 2014. "A review of microfabrication techniques and dielectrophoretic microdevices for particle manipulation and separation". J. Phys. D: Appl. Phys., 47, p. 063001.

[19] Zeinali, S., Cetin, B., Oliaei, S., and Karpat, Y., 2015. "Fabrication of continuous flow microfluidic device with 3D electrode structures for high throughput DEP applications using mechanical machining”. Electrophoresis, 36, pp. 1432-1442.

[20] Canpolat, C., Zhang, M., Rosen, W., Qian, S., and Beskok, A., 2013. "Induced-charge electroosmosis around touching metal rods". J. Fluids Eng., 135(12), March, p. 135.

[21] Green, N. G., Ramos, A., Gonzalez, A., Morgan, H., and Castellanos, A., 2002. "Fluid flow induced by nonuniform ac electric fields in electrolytes on microelectrodes. iii. observation of streamlines and numerical simulation". Physical Review E, 66, August, pp. 026305-1-026305-11.

[22] Gonzalez, A., Ramos, A., Green, N. G., Castellanos, A., and Morgan, H., 2000. "Fluid flow induced by nonuniform ac electric fields in electrolytes on microelectrodes. ii. a linear double-layer analysis". Physical Review E, 61(4), pp. 4019-4028.

[23] Wu, C., Tang, K., Gu, B., Liu, Z., and Wu, Z., 2016. "Concentration-dependent viscous mixing in microfluidics: Modeling and experiments". Microfluid. Nanofluid., 20(6), p. 90 . 